\title{
CORRIGENDUM
}

\section{Childhood obesity: the role of family factors, depressive symptoms and anxiety levels}

I Pinto, L Oliveira, M Pinto, C Calhau and R Coelho

International Journal of Obesity (2011) 35, 1010; doi:10.1038/ijo.2011.109

Correction to: International Journal of Obesity (2011) 35, S161; doi:10.1038/ijo.2011.49

Upon publication of this abstract the lead author noted that the following contributing authors had been omitted from the list of authors - Laura Oliveira, Maria Pinto, Conceição Calhau and Rui Coelho.

The lead author would like to apologise for this error. 